\title{
A DECISION SUPPORT MODEL FOR THE OPTIMAL SITING AND SIZING OF STORAGE UNITS IN STORMWATER DRAINAGE SYSTEMS
}

\author{
M.C. CUNHA ${ }^{1}$, J.A. ZEFERINO ${ }^{1}$, N.E. SIMÕES ${ }^{1}$, G.L. SANTOS ${ }^{2} \&$ J.G. SALDARRIAGA ${ }^{3}$ \\ ${ }^{1}$ MARE, Department of Civil Engineering, University of Coimbra, Portugal. \\ ${ }^{2}$ Department of Civil Engineering, University of Coimbra, Portugal. \\ ${ }^{3}$ CIACUA, Aqueduct and Sewage Research Center, Department of Civil and Environmental Engineering, \\ University of Los Andes, Colombia
}

\begin{abstract}
Climate change and urbanization are the main factors involved in increasing cities' susceptibility to flood events. Extreme rainfall events are occurring more often due to climate change and this, together with the effect of impermeable surfaces, means that the runoff increases. Consequently, the existing stormwater drainage systems need to be adapted. One solution to adapt these systems would be to include infrastructure elements, such as storage units, in the hydraulic network to control flow and reduce peak flows.

This paper presents a decision support model for the optimal siting and sizing of storage units with flow control in existing urban stormwater systems where flood events are frequent. Direct flood damage will be taken into account in the decision process, and thus the cost of construction and maintenance of storage units will be considered along with the cost of flood damage, in the determination of the solution to be implemented. This damage relates to losses in the affected areas and depend on the land use type (e.g. uses of buildings) and on the flood depth affecting it.

A computer program, OptSU, was developed to implement the model. It includes a resolution method based on a simulated annealing algorithm that calls upon a hydraulic simulator whenever necessary. The optimization model is then tested on a case study inspired by a real urban stormwater system in Portugal.

Keywords: detention basins, optimization, urban drainage, urban floods.
\end{abstract}

\section{INTRODUCTION}

Climate change and greater urbanization has led to cities being more susceptible to natural hazards like floods [1]. These natural phenomena endanger people and property and can affect economic activities.

The change in rainfall and surface flow patterns requires an adjustment of the existing urban stormwater drainage systems. One solution to adapt these systems would be to include appropriate structures such as storage units (SUs) in the hydraulic network, to control flow and reduce peak flows.

Given the importance, complexity and high cost of implementing these infrastructure elements, the right techniques must be used to define the best solutions. An efficient drainage system should be able to collect the rainfall runoff and prevent flooding, or at least drastically reduce any damage. The best solution will be the one that minimizes a cost function including both the cost of implementing SUs and the cost of flood damage. This includes damage to the affected buildings, according to their characteristics and the flood depth. 
This paper presents a decision support model for the optimal siting and sizing of SUs with flow control in urban stormwater systems where flood events are frequent. An optimization model has been developed to properly represent the decisions. It embeds discreet variables and nonlinear functions, and simultaneously respects the hydraulic laws and complies with all other relevant regulatory aspects. Therefore a heuristic algorithm is required to solve it [2]. A computer program, OptSU, was developed to implement the model. It includes a resolution method based on a simulated annealing algorithm (SAA) that calls upon a hydraulic simulator whenever necessary. The decision model is then tested on a case study inspired by a real urban stormwater system in Portugal.

\section{METHODOLOGY}

$O p t S U$ is a user-friendly computer program written in Visual Basic, developed to implement the decision support model. The model optimally defines the construction and maintenance costs of SUs, as well as the cost of damage resulting from floods during periods of intense rainfall. The SUs are assumed to be covered concrete structures with vertical walls. An orifice is used to control each SU's downstream flow and allow an efficient flow control throughout the network. The model has inundation and capacity constraints that define limits for floods and for SU dimensions. The behaviour of the drainage network must be consistent with the physical laws of hydrology and hydraulics. Therefore, the variation of the water depths and flows in the drainage network caused by a rainfall event are determined by the widely used hydraulic simulator EPA-SWMM [3]. OptSU runs the SAA for each assessed solution and calls the hydraulic simulator when necessary.

\subsection{Decision model}

This section presents an optimization model for siting and sizing the SUs. Figure 1 shows a schematic representation of an SU implemented in a hydraulic network, including the main variables of the decision model. The decision model is formulated as follows:

$$
\operatorname{Min} \sum_{j \in N_{U}} S_{j}\left(V_{j}\right)+\theta \cdot \sum_{k \in N_{B}} D_{k}\left(h_{k}\right)
$$

subject to:

$$
\begin{gathered}
V_{j}=f\left(Q_{j}^{\text {inf }}, Q_{j}^{\text {out }}\right), j \in N_{U} \\
V_{i}^{\text {surch }}\left(Q_{j}^{\text {inf }}, \phi_{j}\right) \leq V_{\max , i}^{\text {surch }}, i \in N, j \in N_{U} \\
h_{k}=f\left(V_{i}^{\text {surch }}, L_{i}\right), k \in N_{B}, i \in N \\
\sum_{j \in N_{U}} y_{j} \leq N S U \\
V_{j} \leq V_{\max , j} \cdot y_{j}, j \in N_{U} \\
V_{j} \leq V_{\max , j} \cdot y_{j}, j \in N_{U} \\
\phi_{j} \geq \phi_{\max , j} \cdot y_{j}, j \in N_{U} \\
\phi_{j} \leq \phi_{\max , j} \cdot y_{j}, j \in N_{U}
\end{gathered}
$$




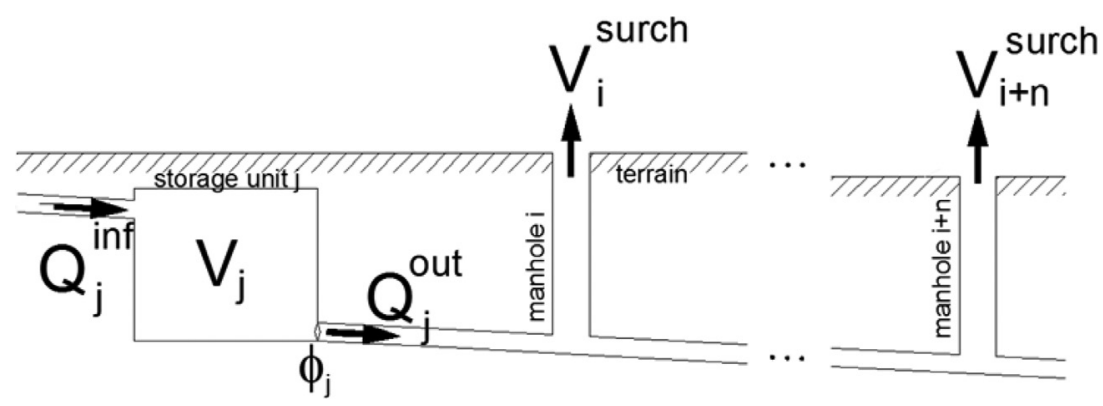

Figure 1: Schematic representation of an SU implemented in the hydraulic network.

$$
\begin{gathered}
Q^{\text {out }} \leq Q_{\max }^{\text {out }} \\
y_{j} \in\{0,1\}, j \in N_{U} \\
V_{i}^{\text {surch }} \geq 0, i \in N
\end{gathered}
$$

where: $\quad N_{U}$ - set of possible locations of SU nodes $j$;

$N-$ set of all nodes $i$;

$N_{B}$ - set of all buildings $k$;

$V_{j}$ - volume of the SU at node $j$;

$S_{j}-$ total cost of the SU at node $j$;

$h_{k}$ - flood depth at building $k$;

$D_{k}$ - flood damage cost at building $k$;

$\theta$ - factor/weight expressing the importance of flooding impacts;

$Q_{j}^{\text {inf }}$ and $Q_{j}^{\text {out }}$ - inlet and outlet flow of the SU at node $j$, respectively;

$V_{i}^{\text {surch }}$ - surcharged volume at node $i$;

$\phi_{j}-\mathrm{SU}$ outlet (orifice) diameter at node $j$;

$L_{i}$ - parameter representing the characteristics of the influence area of node $i$;

$y_{i}$ - binary variable that is one if there is a SU at node $j$ and zero otherwise;

$N S U$ - maximum number of SUs to be implemented in the system;

$Q^{\text {out }}$ - flow at the outfall node;

$Q_{\max }^{\text {out }}$ - maximum flow at the outfall node;

$V_{\min , j}$ and $V_{\max , j}-$ minimum and maximum volume of the SU at node $j$, respectively;

$\phi_{\min , j}$ and $\phi_{\max , j}$ - minimum and maximum SU outlet (orifice) diameter at node $j$, respectively;

$V_{\max , i}^{\text {surch }}$ - maximum local flooding (surcharged) volume at node $i$.

The first term of the objective function (OF) is the total cost of all the implemented SUs. The second term is the total flood damage cost, which is derived from the total damage occurring in all the affected buildings in the study area. The $\theta$ factor in the second term 
allows decision makers to assign the importance of the flooding impacts and enables a better understanding of the tradeoffs between the two terms. This coefficient can also be applied as a penalty to prevent flooding impacts.

Constraint (2) defines the necessary volume of SU at node $j$, resulting from the mass balance between the flow arriving to the SU and the amount of outflow that can reduce downstream flooding. Constraint (3) represents the flood constraints by specifying the limits to the surcharged flows that must be confirmed. A decision maker can allow a certain level of maximum flooding at each node, $i$. The surcharged flood at a downstream node $i$ depends on the inlet flows of the SU at node $j$, and on the respective outlet diameter, which limits the outlet flows of the SU. The calculations associated with constraints (2), and (3) are performed by the hydraulic simulator EPA-SWMM. Constraint (4) defines the inundation depth in each building $k$ that depends on the surcharged volume at node $i$, located in the influence area affecting that building, as well as the characteristics of that area. Constraint (5) limits the number of SUs to a maximum of NSU. Constraints (6) and (7) ensure that the volume at an SU node $j$ is larger than its pre-defined minimum size and smaller than its pre-defined maximum capacity (according to the available space in that location). Constraints (8) and (9) ensure that the outlet diameter at an SU node $j$ is larger than its pre-defined minimum value (very small diameters are likely to become clogged) and smaller than its pre-defined maximum value (that is, the diameter in the downstream conduit). Constraint (10) limits the flow at the outfall node to a maximum of $Q_{\max }^{\text {out }}$. Constraint (11) states that $y_{i}$ are binary variables and (12) represents a non-negativity constraint.

\subsection{Cost functions}

As mentioned above, the OF consists of two terms: the SU construction and maintenance costs and the flood damage costs.

The SU costs can be quantified by means of a function including two terms: a fixed cost, which does not depend on the storage volume of the SU, and a variable cost term, which does. This function can be represented by $S_{j}\left(V_{j}\right)=A+b . V_{j}^{c}$. Parameter $A$ represents the fixed costs, $b$ and $c$ are parameters of the variable cost term. Usually, for this kind of infrastructure element the parameter $c$ takes values less than 1, i.e. the variable cost term expresses an economy of scale. The maintenance costs over the time period can be added to the function as a percentage of the construction cost after being converted to its present value.

Depth-damage curves are the main tools to assess the damage caused by a flood event. These curves are functions that estimate the relation between the inundation depth and the damage caused by the flood event. The damage is given as a percentage of the taxable value of the affected element depending on the flood depth affecting the building. These curves depend on a set of local characteristics of the affected area as well as on the impact characteristics of the flood. Examples of local characteristics are the buildings/land use type, the construction materials and the preparedness of the population for a flood event. The impact characteristics include the inundation depth, flow velocity, duration of inundation and water contamination [4]. These curves $\left(d_{k}\right)$ usually represent damage to the buildings as well as to its contents, depending on the flood depth $\left(h_{k}\right)$ affecting the building. One possible method to determine the damage caused by a flood event is through the product of three amounts: the damage percentage, given by the depth-damage curves, multiplied by the taxable value of the building $\left(e_{k}\right)$ by square meter and by the buildings area $\left(A_{k}\right)$. Therefore, the second term of the OF is calculated by means of the following function: $D_{k}\left(h_{k}\right)=A_{k} \cdot e_{k} \cdot d_{k}\left(h_{k}\right)$. 


\subsection{Solution methods}

OptSU solves the model by using the SAA, which calls on the hydraulic simulator when necessary. Modern heuristics based in nature and physics analogies have become popular in recent years. This technique applies strategies that avoid local optimal solutions [5]. The SAA in particular [6] can be used to solve optimization models involving complex systems. The SAA has been used in many models for hydraulic systems planning, with notable results $[7,8]$.

A typical SAA run consists of a number of successive (random) neighbourhood moves in the problem search space. The SAA is controlled by a set of parameters, of which the most important is the temperature. Initially, when the temperature is high, a large variety of candidate solutions can be accepted. As the temperature falls during the process, according to a predefined cooling schedule, there is less likelihood of accepting poor solutions. Starting from an initial solution, the SAA progressively searches for new candidate solutions until the stopping criterion is met.

In each SAA assessed solution, the information regarding the hydraulic network behaviour is exchanged between EPA-SWMM and the optimization algorithm through a toolkit [9]. $O p t S U$ accesses and modifies the network data, for example the generation of new candidate solutions, according to the SAA.

\section{CASE STUDY}

The proposed methodology was tested on a case study inspired on a real network from an urban area in Portugal. The case study demonstrates and evaluates the behaviour of the model based on cost minimization. The region has a total area of 16.6 ha. Based on IDF curves, the alternating block method was used to obtain a 2 hours design storm with a return period of 100 years.

The cost of the SUs was estimated using a function determined by Wiegand et al. [10]. It was also assumed that the annual maintenance cost is a percentage of the construction cost and, according to Narayanan and Pitt [11], they are between 3 and 5\% of the total construction cost. Therefore, taking the maintenance cost as $3 \%$ of the total construction cost, an interest rate of $5 \%$ and a time period of 40 years, the function giving the present value of the SU total costs for the year of 2015 is represented in Fig. 2.

The damage is evaluated with three depth-damage curves, one for each building type: residential, commercial and public facilities. The taxable unit value by square meter is an average of the existing buildings for each land use type. Figure 3 represents the product between each land use type curve and its respective taxable value. These functions consider the damage to both the affected buildings and their contents, according to the flood depth in each building.

The reference situation (the current situation with no SUs implemented), has a total flooding of 2,416 $\mathrm{m}^{3}$ in the nodes of the system, amounting to a total damage cost of 2.55 million euros. The study area and the flood depths in each influence area are shown in Fig. 4.

Theoretically, an SU could be implemented at any point in the network and then the optimization model would provide a solution based on the specific goals of the problem. However, this would result in a very complex and computationally demanding problem. A pre-evaluation of the existing network and the reference situation can lead to the number of potential SU locations being reduced. This evaluation should consider the space available and feasibility of implementing SUs.

For this case study, after analysing the urban characteristics and the reference situation in terms of flooding, six possible locations for the implementation of SUs were defined (Fig. 5). 


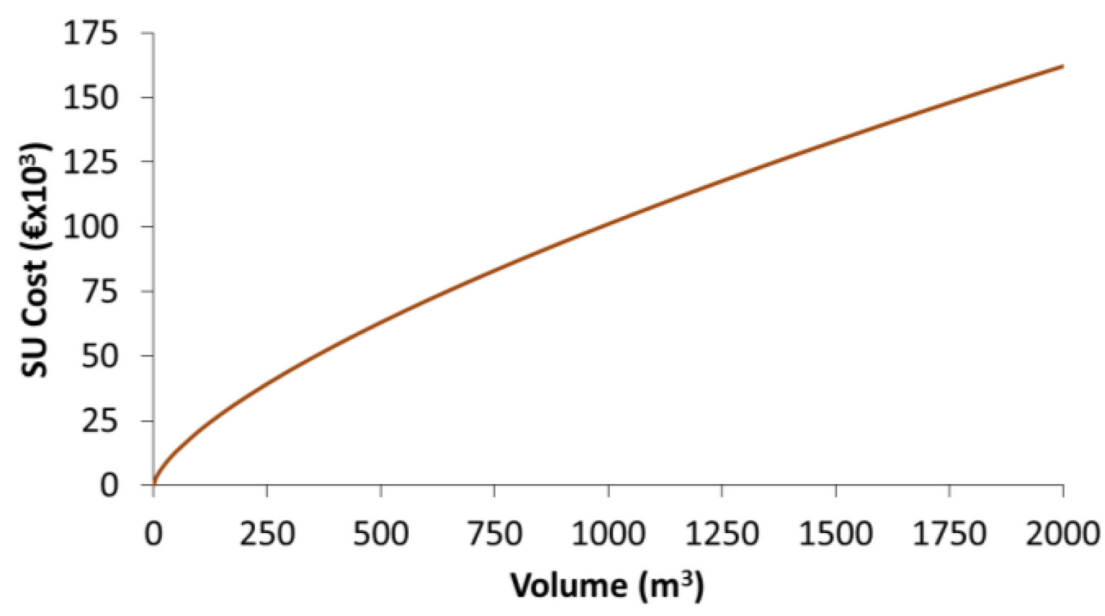

Figure 2: SU construction and maintenance cost function.

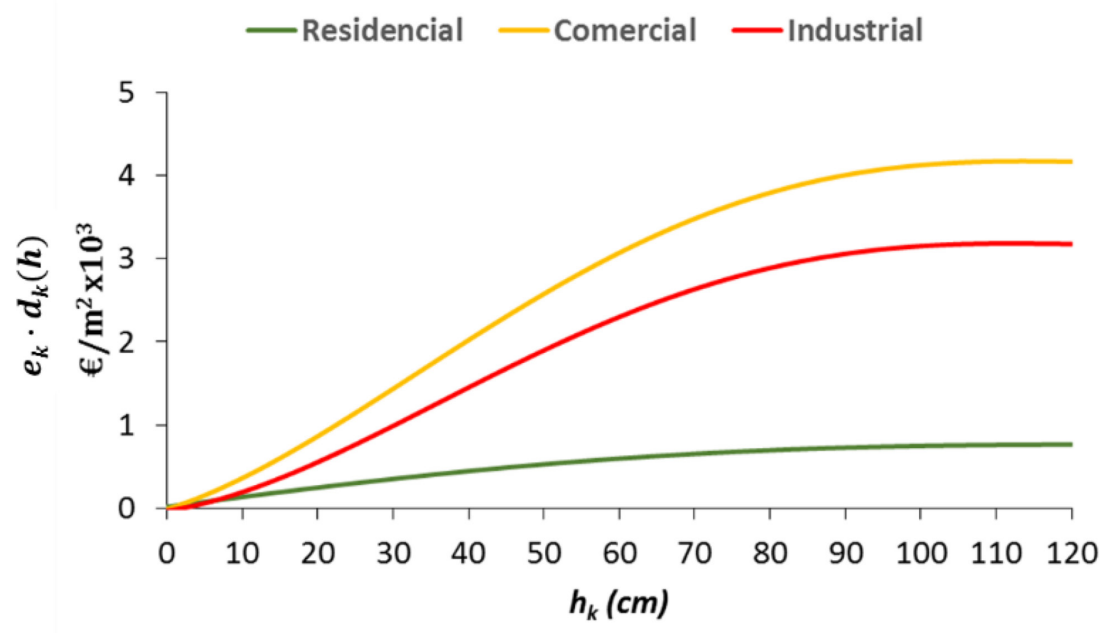

Figure 3: Depth-damage curves for each land use type.

The minimum volume of each SU was assumed to be $100 \mathrm{~m}^{3}$ and the maximum volume $2,000 \mathrm{~m}^{3}$. The outlet downstream of a SU can have a minimum diameter of $25 \%$ of the maximum diameter of the downstream conduit cross section.

Two examples were designed for the case study. They differ in the importance given to the flood damage costs (weight factor $-\theta$ ). The weight factor for flood damage can vary, for example, from 1 (important) to 5 (very important), thus showing how can it affect the results. Therefore, the examples are: Example A, with $\theta=1$; Example $\mathrm{B}$, with $\theta=5$.

\subsection{Results}

Table 1 summarizes the SUs' results (location, volume, outlet diameter and cost), flood results (volume and damage expenses), and the total cost for each example. 


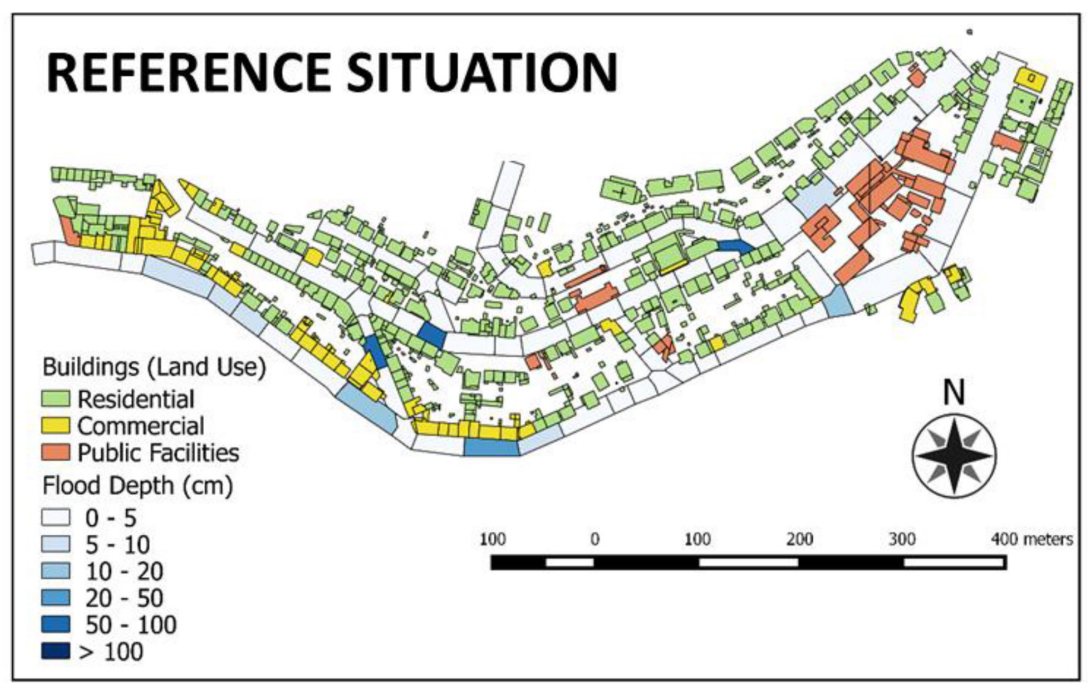

Figure 4: Flood depths for the reference situation.

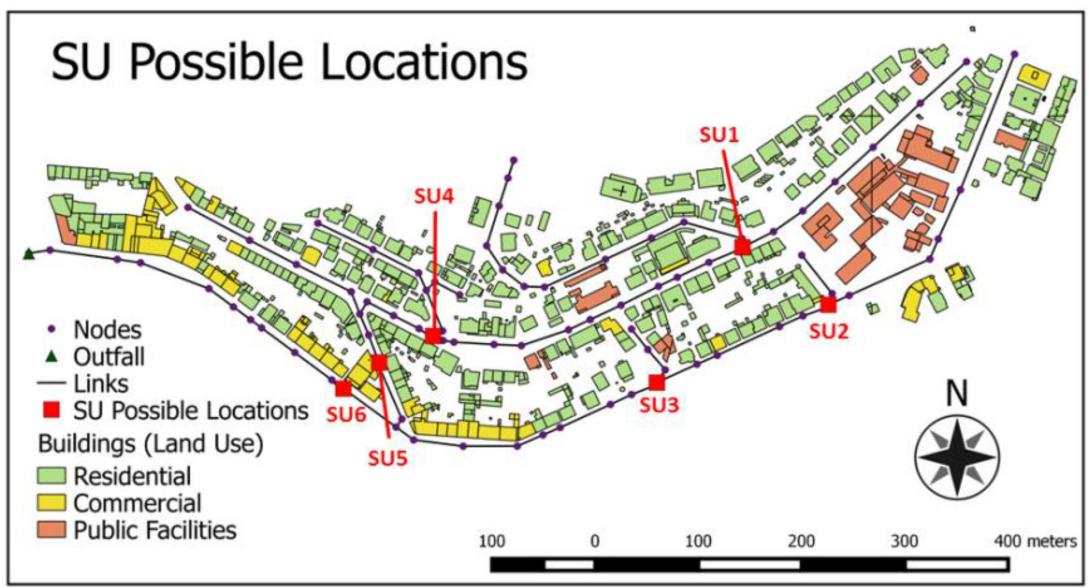

Figure 5: Possible sites to implement SUs.

Flooding volumes and total cost reductions of $71 \%$ and $84 \%$, respectively, (98\% of damage cost reduction) were found for Example A. These figures represent significantly lower costs than those of the reference situation. That is because the optimization model not only reduces flooding volumes, it also prevents inundations in influence areas that would affect more buildings (or those with a higher unit value). Therefore, it sites the rest of flooding volumes in nodes away from those influence areas. Figure 6 represents the flood depths for Example A, where it is possible to see that the depths are much lower than in the reference situation. It can be noted that the flooded areas only affect a few buildings and none of them has commercial use (which have the highest unit values).

Flooding volumes and total cost reductions of $72 \%$ and $82 \%$, respectively, (99\% of damage cost reduction) were found for Example B. Flooding volumes fell $72 \%$ relative to the reference situation. The majority of the SUs have bigger dimensions than Example A, with 
Table 1: Results for the two examples and the reference situation.

\begin{tabular}{|c|c|c|c|c|c|c|c|c|c|c|c|}
\hline & \multirow{2}{*}{$\mathrm{SU}_{1}$} & \multirow{2}{*}{\multicolumn{2}{|c|}{$\mathrm{SU}_{2} \mathrm{SU}_{3}$}} & \multirow{2}{*}{$\mathrm{SU}_{4}$} & \multirow{2}{*}{$\mathrm{SU}_{5}$} & \multirow{2}{*}{$\mathrm{SU}_{6}$} & \multirow{2}{*}{$\Sigma \mathbf{S U}$} & \multicolumn{3}{|c|}{$\Sigma_{\mathrm{i}} \mathbf{V}_{\mathrm{i}} \quad \Sigma_{\mathrm{k}} \mathbf{D}_{\mathrm{k}}$ Total } \\
\hline & & & & & & & & & $\left(\mathrm{m}^{3}\right)$ & $(€ \mathrm{X}$ & $\left.10^{3}\right)$ \\
\hline \multirow{3}{*}{$\begin{array}{l}\text { Reference } \\
\text { Situation }\end{array}$} & SU Vol. $\left(\mathrm{m}^{3}\right)$ & - & - & - & - & - & - & 0 & \multirow{3}{*}{2,416} & \multirow{3}{*}{2,547} & \multirow{3}{*}{2,547} \\
\hline & Orif. Diam. (mm) & - & - & - & - & - & - & & & & \\
\hline & SU Cost $\left(€ \times 10^{3}\right)$ & - & - & - & - & - & - & 0 & & & \\
\hline \multirow{3}{*}{ Example A } & SU Vol. $\left(\mathrm{m}^{3}\right)$ & 1,282 & - & 630 & 868 & 668 & - & 3,448 & \multirow{3}{*}{710} & \multirow{3}{*}{54} & \multirow{3}{*}{413} \\
\hline & Orif. Diam. (mm) & 351 & - & 266 & 429 & 464 & - & & & & \\
\hline & SU Cost $\left(€ \times 10^{3}\right)$ & 119 & - & 73 & 91 & 76 & - & 359 & & & \\
\hline \multirow{3}{*}{ Example B } & SU Vol. $\left(\mathrm{m}^{3}\right)$ & 2,000 & - & 728 & 1,704 & 100 & 100 & 4,632 & \multirow{3}{*}{668} & \multirow{3}{*}{39} & \multirow{3}{*}{466} \\
\hline & Orif. Diam. (mm) & 253 & - & 252 & 290 & 500 & 800 & & & & \\
\hline & SU Cost $\left(€ \times 10^{3}\right)$ & 161 & - & 81 & 144 & 21 & 21 & 427 & & & \\
\hline
\end{tabular}

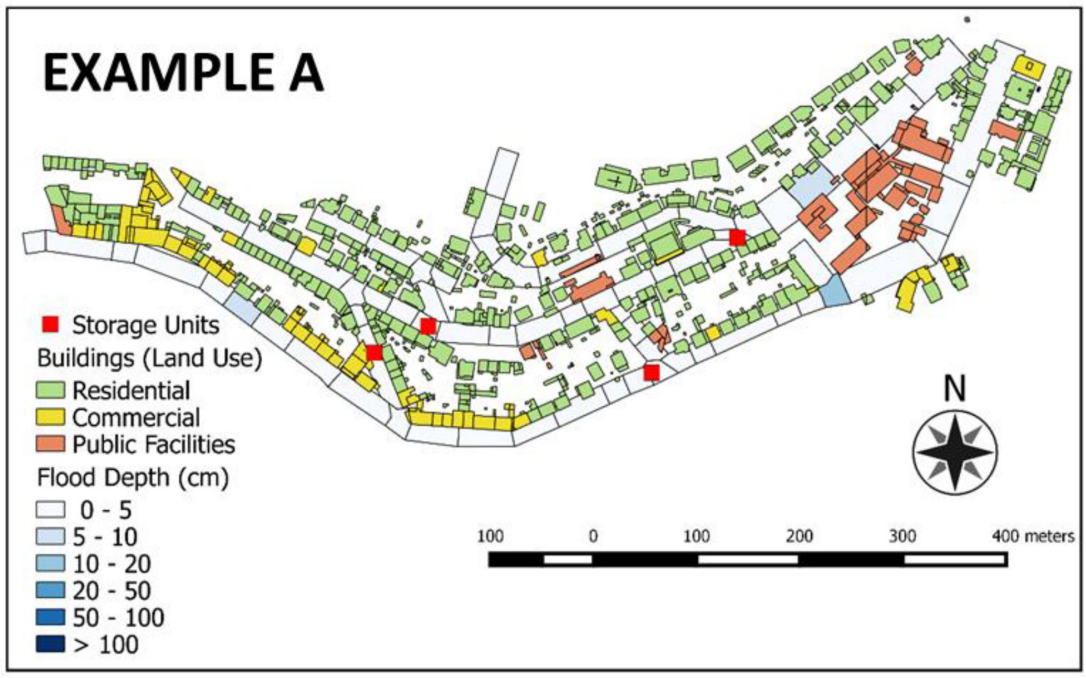

Figure 6: Flood depths in Example A.

SU1 having the maximum volume allowed. SU5, the only one smaller than those in Example A, has the minimum volume allowed since in this example bigger volumes are stored in the upstream SUs and in addition there is the downstream SU6, also with the minimum volume. Even though SU5 and SU6 have the minimum volume and the maximum allowed orifice diameters, a closer look at the results show that their storage volumes are important since they reach their maximum storage at some point during the runoff.

In terms of flood depths, Example B (Fig. 7) is very similar to Example A, even though the total flooding volume is slightly lower. The difference between the examples is relatively small but it is still possible to understand the effects of the different weight factors assigned 


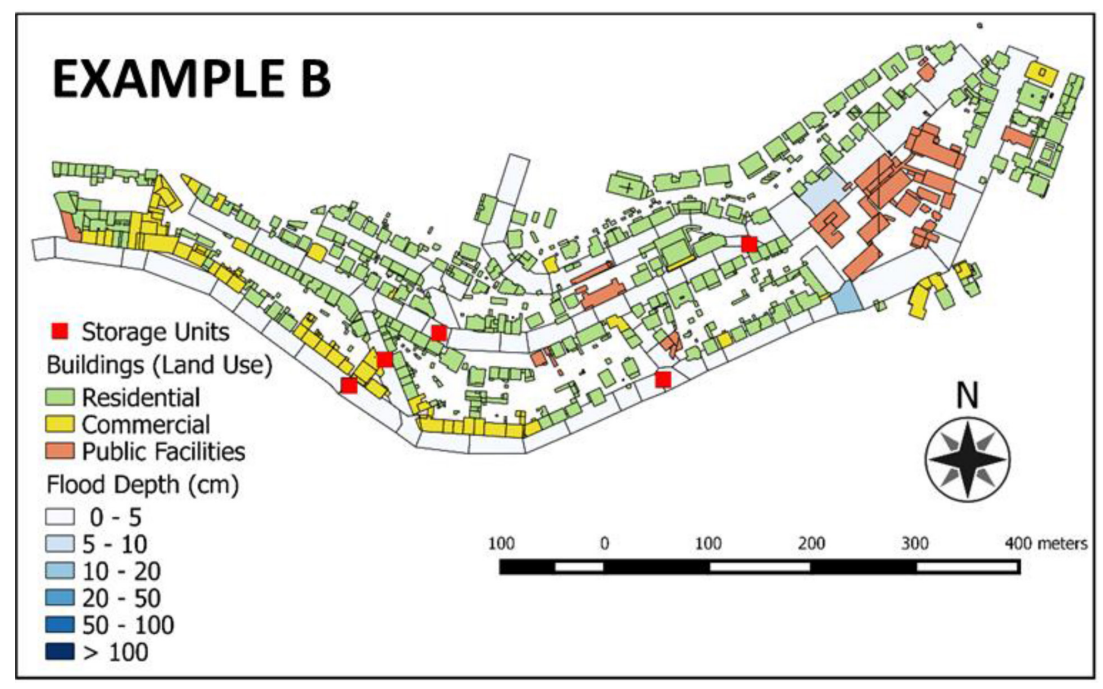

Figure 7: Flood depths in Example B.

to the flood damage costs. The construction of SU6 eliminates the flooding volume in one of the flooded influence areas of Example A. Although the total storage volume in Example B is $34 \%$ greater than in Example A, the total cost of the SUs is only 19\% higher. That is because of the economies of scale effect. In contrast, the damage cost in Example B is 28\% lower than in Example A. Because of that, and since the weight factor is higher, Example B results in a solution with lower damage costs than Example A, but which is more expensive overall $(13 \%)$. Then, it is up to the decision maker to decide if the reduction in flood damage economic and socially offsets the increase in the SUs' construction cost.

However, these examples show that increasing the damage cost weight factor does not significantly reduce the inundations. This is probably related to the possible sites considered for the SUs, since the influence areas where the flooding occurs are upstream of them. However, the implementation of SUs in those upstream nodes is not viable due to local constraints. SU2 is not used in either example since its node and its downstream nodes are located in influence areas where the flooding volumes do not affect any building, thus causing no damage. Therefore, there is no need to reduce the flooding volume there. Moreover, SU3 is located slightly downstream of SU2.

\section{CONCLUSIONS}

This article describes a decision support model and its computational application, OptSU, for the optimal siting and sizing of SUs in urban stormwater systems. The SAA and the EPASWMM hydraulic simulator were used to solve the model. OptSU has been tested on a case study based on a real urban stormwater system in Portugal by applying the methodology to two examples that varied only in the flood damage cost weight factor.

As expected, both examples had a large reduction of the flooding volumes, in particular regarding the flooding damage, and thus the total cost. However, they had different reductions. In Example A, assigning the same importance to flood damage and to SU costs led to a total cost reduction of $84 \%$. In Example B the total cost reduction was slightly lower (82\%). Increasing the weight factor results in the construction of larger SUs and consequently in a higher total cost, even with a greater reduction of the flood damage than Example A. Nev- 
ertheless, the model was efficient at finding minimum cost solutions that still have small flooding volumes but do not cause any significant damage. Both examples almost totally eliminated the damage in the reference situation (98\% in Example A and 99\% in Example B).

The cost functions considered for the construction and maintenance of SUs and for the damage cost estimation were based on literature functions for situations similar to the case study. In a real application, these curves should be determined for the specific study area. Regarding the depth-damage functions, it is important to note that since it is difficult to estimate all the possible damage and influencing parameters, this case study considered only the flooding depth affecting each building and the building/land use type.

Further developments of this study look at problems of climate change and the increase in urban development in order to obtain robust solutions that work properly in all possible scenarios.

\section{ACKNOWLEDGMENTS}

The authors would like to thank Mexichem, Colombia for supporting this research through the project "Drenaje Urbano y Cambio Climático: hacia los sistemas de alcantarillado del futuro. Fase II", without which it would not be possible to conclude the work.

\section{REFERENCES}

[1] Butler, D. \& Davies, J.W., Urban Drainage, 3rd edn., CRC Press: London, 2011.

[2] Maier, H., Kapelan, Z., Kasprzyk, J., Kollat, J., Matott, L., Cunha, M., Dandy, G., Gibbs, M., Keedwell, E., Marchi, A., Ostfeld, A., Savic, D., Solomatine, D., Vrugt, J., Zecchin, A., Minsker, B., Barbour, E., Kuczera, G., Pasha, F., Castelletti, A., Giuliani, M. \& Reed, P., Evolutionary algorithms and other metaheuristics in water resources: current status, research challenges and future directions. Environmental Modelling and Software, 62, pp. 271-299, 2014.

http://dx.doi.org/10.1016/j.envsoft.2014.09.013

[3] Rossman, L., Storm water management model user's manual, version 5.0. Cincinnati: National Risk Management Research Laboratory, Office of Research and Development, US-EPA, 2010.

[4] Merz, B., Kreibich, H., Schwarze, R. \& Thieken, A., Review article "Assessment of economic flood damage". Natural Hazards and Earth System Sciences, 10(8), pp. 1697-1724, 2010.

http://dx.doi.org/10.5194/nhess-10-1697-2010

[5] Rothlauf, F., Design of Modern Heuristics: Principles and Application, Springer Science \& Business Media: Berlim, 2011.

[6] Kirkpatrick, S., Gellatt, C. \& Vecchi, M., Optimization by simulated annealing. Science, 220(4598), pp. 671-680, 1983. http://dx.doi.org/10.1126/science.220.4598.671

[7] Zeferino, J.A., Antunes, A.P. \& Cunha, M.C., An efficient simulated annealing algorithm for regional wastewater system planning. Computer-Aided Civil and Infrastructure Engineering, 24(5), pp. 359-370, 2009.

http://dx.doi.org/10.1111/j.1467-8667.2009.00594.x

[8] Marques, J., Cunha, M. \& Savić, D.A., Multi-objective optimization of water distribution systems based on a real options approach. Environmental Modelling and Software, 63, pp. 1-13, 2015.

http://dx.doi.org/10.1016/j.envsoft.2014.09.014 
[9] Iglesias, P.L. \& Martínez, F.J., Guía de Manejo Rápido de la Librería de Conexión de SWMM 5, Universidad Politécnica de Valencia, España, 2014.

[10] Wiegand, C., Schueler, T., Chittenden, W. \& Jellick, D., Cost of urban runoff quality controls. In Urban Runoff Quality - Impact and Quality Enhancement Technology, eds. B. Urbonas \& L.A. Roesner, pp. 366-382. American Society of Civil Engineers: New York, NY, 1986.

[11] Narayanan, A. \& Pitt, R., Costs of Urban Stormwater Control Practices, The University of Alabama, United States of America, 2006. 